\title{
Multiplexing of spatial modes in the mid-IR region
}

Gailele, Lucas, Maweza, Loyiso, Dudley, Angela, Ndagano, Bienvenu, Rosales-Guzman, Carmelo, et al.

Lucas Gailele, Loyiso Maweza, Angela Dudley, Bienvenu Ndagano, Carmelo Rosales-Guzman, Andrew Forbes, "Multiplexing of spatial modes in the midIR region," Proc. SPIE 10090, Laser Resonators, Microresonators, and Beam Control XIX, $100900 Z$ (20 February 2017); doi: 10.1117/12.2256085

SPE. Event: SPIE LASE, 2017, San Francisco, California, United States 


\title{
Multiplexing of spatial modes in the mid-IR region
}

\author{
Lucas Gailele ${ }^{\mathrm{a}, \mathrm{b}}$, Loyiso Maweza ${ }^{\mathrm{b}}$, Angela Dudleya,b, Bienvenu Ndagano ${ }^{\mathrm{a}}$, Carmelo \\ Rosales-Guzman ${ }^{\mathrm{a}}$, and Andrew Forbes ${ }^{\mathrm{a},{ }^{*}}$ \\ aUniversity of the Witwatersrand, School of Physics, Johannesburg, Wits 2050, South Africa \\ ${ }^{\mathrm{b} C S I R}$ National Laser Centre, Pretoria 0001, PO Box 395 South Africa
}

\begin{abstract}
Traditional optical communication systems optimize multiplexing in polarization and wavelength both transmitted in fiber and free-space to attain high bandwidth data communication. Yet despite these technologies, we are expected to reach a bandwidth ceiling in the near future. Communications using orbital angular momentum (OAM) carrying modes offers infinite dimensional states, providing means to increase link capacity by multiplexing spatially overlapping modes in both the azimuthal and radial degrees of freedom. OAM modes are multiplexed and de-multiplexed by the use of spatial light modulators (SLM). Implementation of complex amplitude modulation is employed on laser beams phase and amplitude to generate Laguerre-Gaussian (LG) modes. Modal decomposition is employed to detect these modes due to their orthogonality as they propagate in space. We demonstrate data transfer by sending images as a proof-of concept in a lab-based scheme. We demonstrate the creation and detection of OAM modes in the mid-IR region as a precursor to a mid-IR free-space communication link.
\end{abstract}

Keywords: Orbital Angular Momentum, Modal Decomposition, Free-Space Communication, Multiplexing, Complex Amplitude Modulation.

*Andrew Forbes, andrew.forbes@wits.ac.za

\section{INTRODUCTION}

The race to achieve high speed data transfer both in fibers and free-space has long been pursued since the beginning of the 21st century, with the ever increasing data usage such as social media sites like Facebook and Youtube to stream high definition videos. Optical fiber communication is predicted to reach its peak due to a fundamental data ceiling caused by nonlinear effects in fibers. ${ }^{1}$ Further more, optical fibers are very expensive to buy and install and most inner city buildings are not connected to data connections. Free-space optics ( or sometimes known as wireless optical communication ) provides a solution to this long known problem called the lastmile and also it provides an efficient replacement to optical fiber and predicted to also offer data connection at very high speeds and at a cost effective price. Forty years have gone by where free-space optical communication technologies have developed to its full potential having a multitude of applications ranging from satellite communication, ${ }^{2}$ telecommunication ${ }^{3}$ just to name a few. Free-space communication has been performed typically using visible light over a turbulent media. ${ }^{4-8}$ Here we demonstrate creation and detection of spatial modes with high fidelity. We briefly describe and employ complex amplitude modulation and modal decomposition to the later and we then multiplex spatial modes and send pictures in free-space.

\section{METHODS FOR MANIPULATING MID-IR LIGHT}

\subsection{Creating spatial modes by complex amplitude modulation}

The development of SLMs provides a means to digitally manipulate light into different shapes and sizes without using physical and expensive diffraction optical elements that manipulate light into one shape. The main objective of the complex amplitude modulation technique is to transform information of a optical field in terms of its phase and amplitude into a phase-only function encoded on a SLM according to: ${ }^{9}$

$$
U_{a}=T U_{b}, \quad T=\frac{U_{a}}{U_{b}} e^{i\left(\phi_{a}-\phi_{b}\right)} \rightarrow e^{i \Omega_{S L M}}
$$

Laser Resonators, Microresonators, and Beam Control XIX, edited by Alexis V.

Kudryashov, Alan H. Paxton, Vladimir S. Ilchenko, Proc. of SPIE Vol. 10090, $100900 Z$

(C) 2017 SPIE · CCC code: $0277-786 X / 17 / \$ 18 \cdot$ doi: $10.1117 / 12.2256085$

Proc. of SPIE Vol. 10090 100900Z-1 
where $\Omega_{S L M}$ is a real valued function and within the range $[0,2 \pi]$. Arrizon ${ }^{10}$ demonstrated how we can perform complex amplitude modulation on a phase-only SLM by employing a transmission function of the form:

$$
h(x, y)=e^{i \psi(a, \phi)},
$$

Where the function $\psi(a, \phi)=f(a) \sin (\phi)$, correctly encodes the phase modulation we desire (LG modes). Fig $2(\mathrm{a}, \mathrm{b}, \mathrm{c}, \mathrm{d})$ is showing the type of holograms we get when we encode the transmission function (which performs a phase modulation)according to Eq 2. The corresponding images in Fig 2(e,f,g,h) are the theoretical results of LG modes that correspond to its hologram which can be achieved by illuminating the SLM with a beam with a flat wavefront.

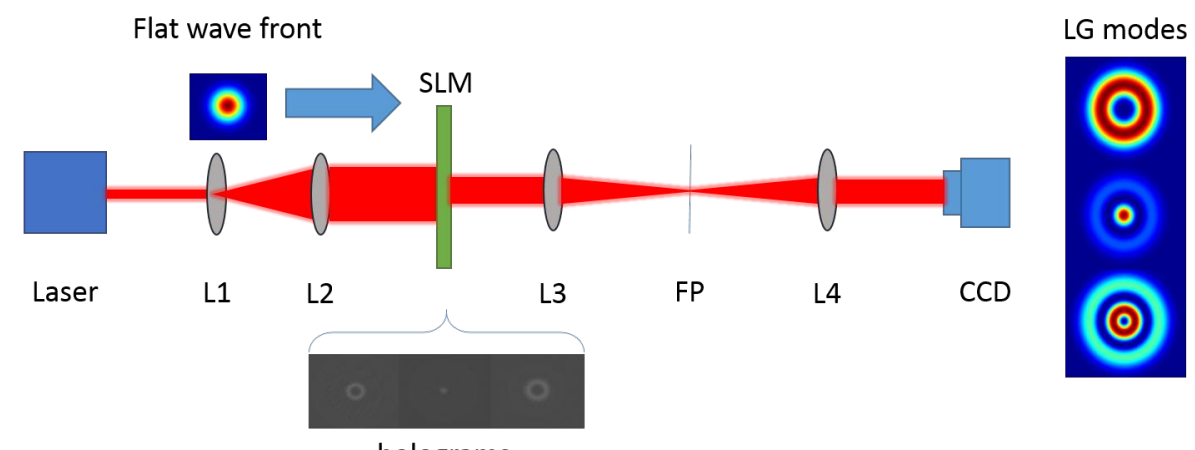

holograms

Figure 1. Experimental setup: Creation of LG modes entails the use of a laser beam, lenses and a SLM addressed with various holograms. The SLM is illuminated with horizontally polarized light and addressed with holograms that modulate amplitude and phase. L1-L2 sets up a Galilean telescope, and the 4f system made up of L3-L4 transfers the phase (L3) and the amplitude (L4) and images the field onto the CCD camera.

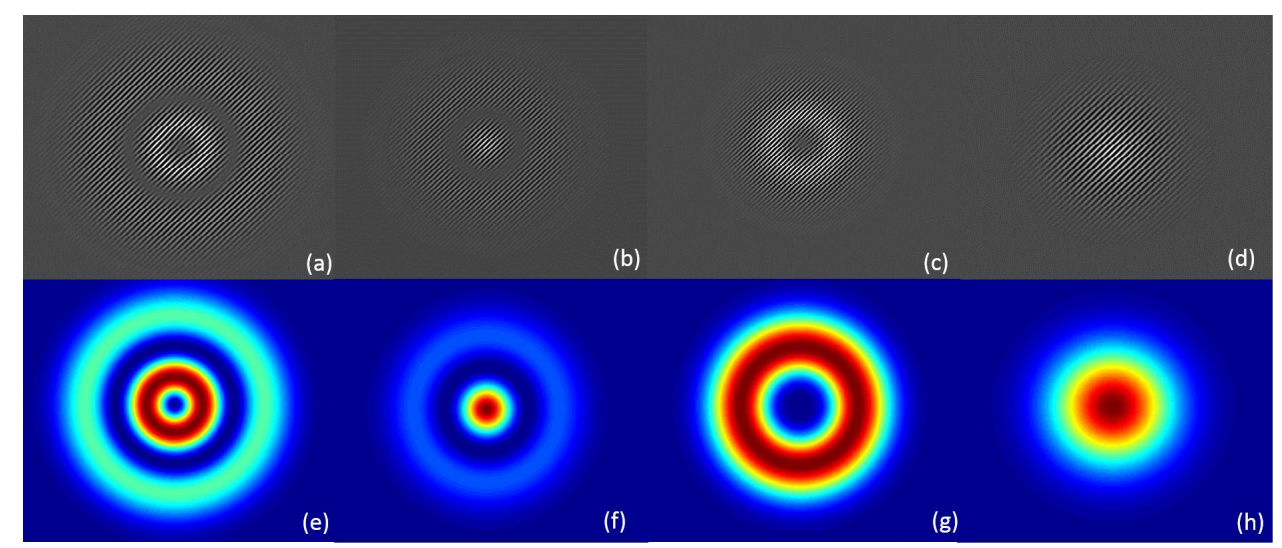

Figure 2. Typical complex amplitude modulation holograms (a,b,c,d) used to address SLM's and their corresponding theoretical intensity distribution of LG modes of different orders (e,f,g,h). 


\subsection{Detection of spatial modes by modal decomposition}

Modal decomposition has long been known and previously applied to pattern recognition ${ }^{11}$ and also most recently to wavefront reconstruction. ${ }^{12,13}$ With the aid of SLMs and digital holograms the characterization of laser beams by finding its beam quality $M^{2}$, phase and wavefront with very high fidelity can be achieved. ${ }^{13-15}$ The main idea behind modal decomposing optical fields is to take an unknown optical field (the mode to be detected) and express it as a linear combination of orthogonal basis functions: ${ }^{16}$

$$
U(\vec{r})=\sum_{l=1}^{\infty} \alpha_{l} \psi_{l}(\vec{r})
$$

where $\vec{r}=(x, y)$ is a spatial coordinate in rectangular form, $\alpha_{l}=\sigma_{l} e^{i \Delta \phi_{l}}$ is called the complex expansion coefficient with amplitude given by $\sigma_{l}$. The inter-modal phase $\Delta \phi_{l}=\phi_{l}-\phi_{0}$ measured with respect to a reference mode (typically chosen as the fundamental Gaussian mode). Calculation of these complex expansion coefficients $\sigma_{l}$ is the main objective. Employing digital holograms with the aid of an SLM and a Fourier transforming lens, we can perform an inner-product measurement to extract the complex coefficients. The displayed holograms act as a match-filter to the incoming optical field, given by:

$$
T_{l}(\vec{r})=\psi_{l}^{*}(\vec{r}),
$$

where the asterisk "*" signifies the complex conjugate. From this transmission function we can measure the optical field intensity $I_{l}$ at the optical axis of the Fourier plane and this intensity is directly proportional to $\sigma_{l}^{2}$. In order to determine the inter-modal phase difference we employ two transmission functions where each

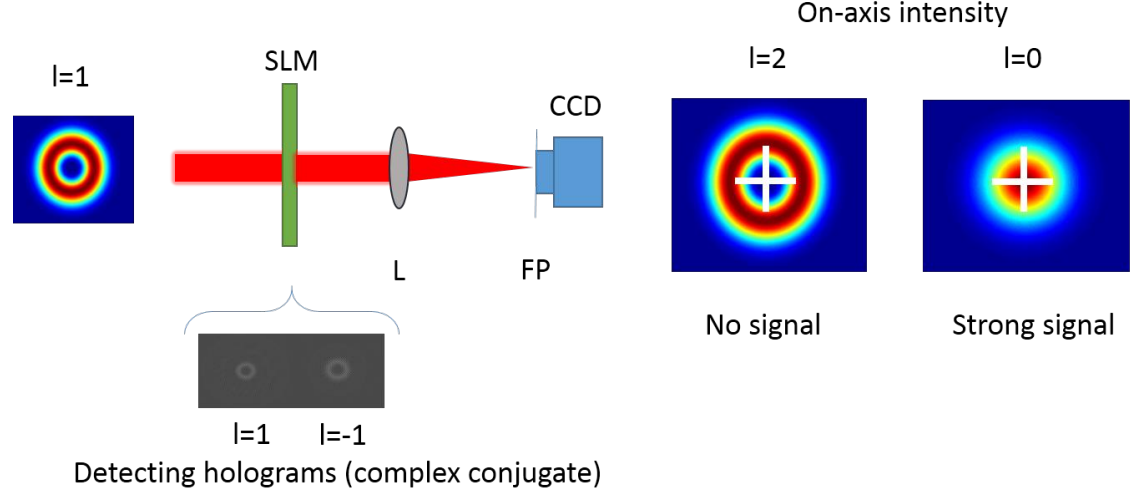

Figure 3. Experimental setup: An incoming LG mode with topological charge $l=1$ and radial index $p=0$ is modally decomposed by the SLM addressed by detecting holograms. At the Fourier plane the on-axis intensity is detected by the CCD camera.

transmission function represents an interferometric superposition of the field given by:

$$
\begin{aligned}
& T_{l}^{\cos }=\left[\psi_{0}^{*}(\vec{r})+\psi_{l}^{*}(\vec{r})\right] / \sqrt{2}, \\
& T_{l}^{s i n}=\left[\psi_{0}^{*}(\vec{r})+i \psi_{l}^{*}(\vec{r})\right] / \sqrt{2},
\end{aligned}
$$

By performing and inner product measurement of the unknown optical field with the transmitting functions given by Eq (5), the stemming intensities in the optical Fourier plane will be given by:

$$
\begin{aligned}
& I_{l}^{\cos } \propto \sigma_{0}^{2}+\sigma_{l}^{2}+2 \sigma_{0} \sigma_{l} \cos \Delta \phi_{l}, \\
& I_{l}^{s i n} \propto \sigma_{0}^{2}+\sigma_{l}^{2}+2 \sigma_{0} \sigma_{l} \sin \Delta \phi_{l},
\end{aligned}
$$

From these measurement the phases can be calculated without any ambiguity according to:

$$
\Delta \phi_{l}=-\arctan \left[\frac{2 I_{l}^{s i n}-\sigma_{l}^{2}-\sigma_{0}^{2}}{2 I_{l}^{c o s}-\sigma_{l}^{2}-\sigma_{0}^{2}}\right]
$$


It is also possible to do a modal decomposition measurement by performing a single inner-product measurement by introducing a spatial carrier frequency $\vec{F}(r)$ which introduces a spatial separation of intensities in the optical Fourier plane: ${ }^{8}$

$$
T(\vec{r})=\sum_{l=1}^{\infty} T_{l}(\vec{r}) e^{i \vec{F}_{l} \vec{r}}
$$

By employing this transmission function we can then multiplex holograms and measure the amplitudes and phase simultaneously.

\section{RESULTS}

We demonstrate, in both the VIS and Mid-IR regions with complex amplitude modulation, that LG modes can be created and detected via modal decomposition with high fidelity. A beam with a flat wavefront was illuminated the SLM which is addressed with a digital hologram performing complex amplitude modulation. The intensity distribution showing different orders of LG modes was observed and recorded by the CCD camera as shown in Fig 4(a) in the visible region and also in Fig 4(b) in the Mid-IR region. After creation of LG modes we then employed modal decomposition on these beams in both wavelength regions. In Fig 4(c) modal decomposition was performed with the radial index $p=0$ and the azimuthal index being varied in the range $l=(-5,5)$. Then we also did a modal decomposition with $p=l=1$ with different values of radial index $p$ and azimuthal index $l$ as shown by Fig 4 (d). Spatial multiplexing was conducted using both the visible (532nm) and Mid-IR (2053nm) wavelengths, this required the use of two SLMs with different coatings but the principle is still the same. Now that we are able to detect and create the modes information was transfered by sending a 100x100 gray scale picture of Maxwell, was sent pixel by pixel over free-space. The space between the modes was chosen to be different to minimize the cross-talk between closely related modes as shown in Fig 5(a). Different shades of gray

(a)
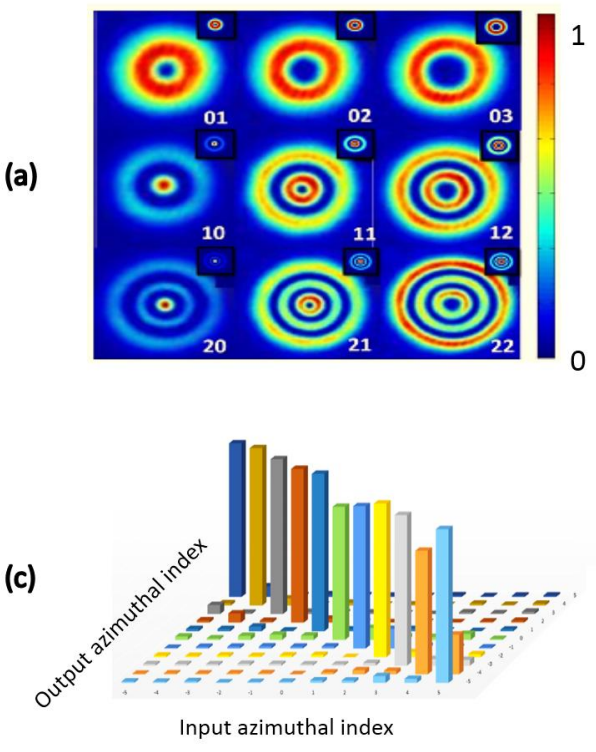

(b)

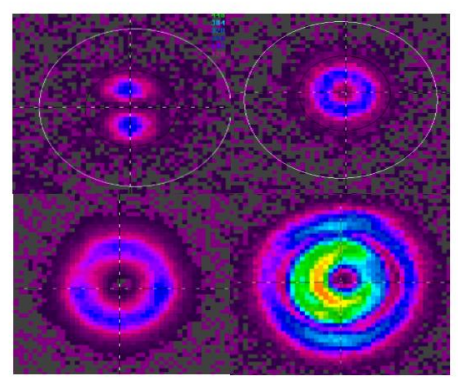

(d)

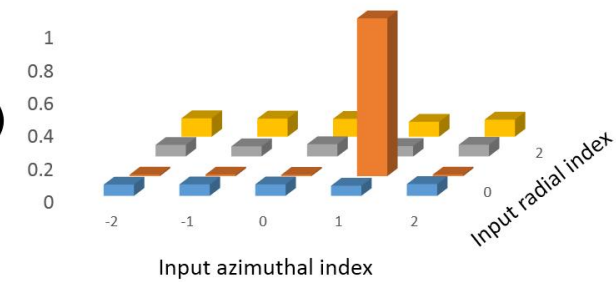

Figure 4. (a) LG modes generated using a 532nm beam indices $l$ and $p$, (b) the LG modes generated using a 2053nm beam. (c) Results obtained from modal decomposition of LG modes with varying $l$ and $p=0$ and (c) modal decomposition of LG mode with $l=1$ while $p=0$ where both intensities in (c) and (d) are normalized to unity.

were chosen and matched with a particular spatial mode of light. These modes were sent through free-space pixel by pixel and they were then matched with a detecting hologram multiplexed with holograms that are inverses of holograms used to create the modes (complex conjugates). At the Fourier plane the modes will be spatially 
separated to different location as shown in Fig 5(b) due to the multiplexed holograms having different spatial carrier frequencies according to Eq 8.

(a)
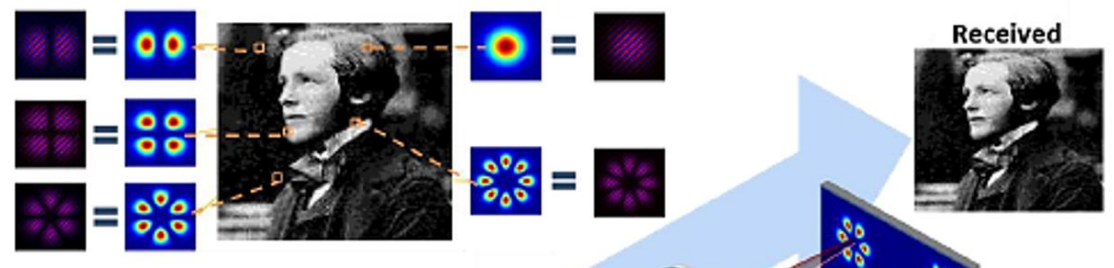

(b)
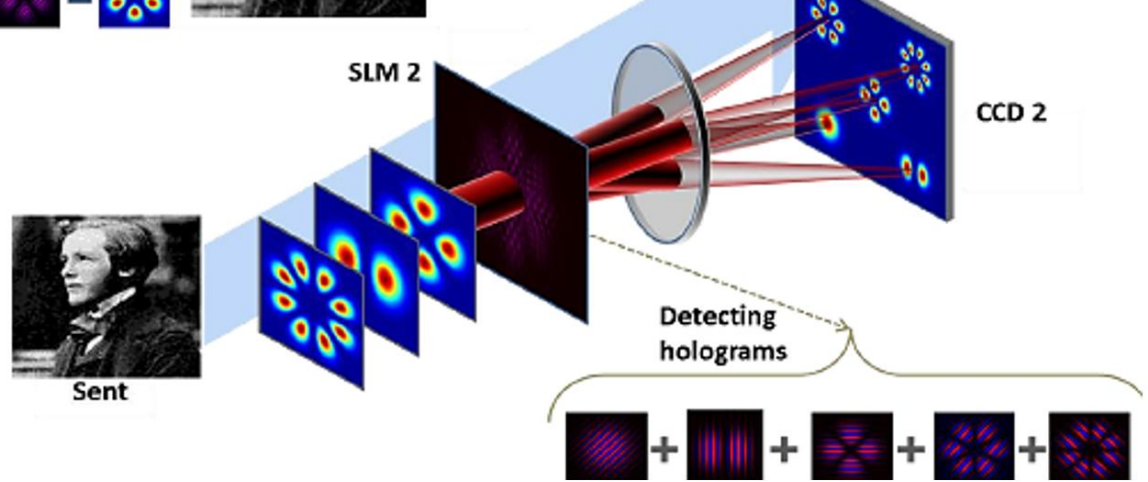

Figure 5. Information transfer using spatial modes of light. (a) Information encoding using spatial modes which are matched with a shade of gray. (b) Sending information in free-space and detected by the multiplexed holograms addressing the SLM. These detected modes will be spatially separated in the Fourier plane where signals will be read off by the CCD camera.

\section{MID-IR FREE-SPACE COMMUNICATION}

It is desirable to have an optical link that has minimal attenuation from atmospheric absorption, scattering and turbulence. The atmosphere is made up of different layers and it is over the troposphere where most gases and particles are concentrated. Optical field scattering can be divided into two phenomenon: Rayleigh scatteringby particles in the atmosphere that are small when compared to the wavelength of the optical field and Mie scattering- by atmospheric particle that are comparable to the wavelength of the optical field. However for wavelengths that are much larger than atmospheric particles we get no scattering. With this fact it is the first motivation behind the choice of a using 2 micron wavelength source. Another factor that we took into consideration was absorption. Air molecules such as carbon dioxide $\mathrm{CO}_{2}$, water vapor $\mathrm{H}_{2} \mathrm{O}$, ozone $\mathrm{O}_{3}$ and nitrogen dioxide $\mathrm{NO}_{2}$ are most responsible for the optical field energy absorption. ${ }^{17}$ In the mid-IR wavelengths these molecules will be affecting to radiation absorption.

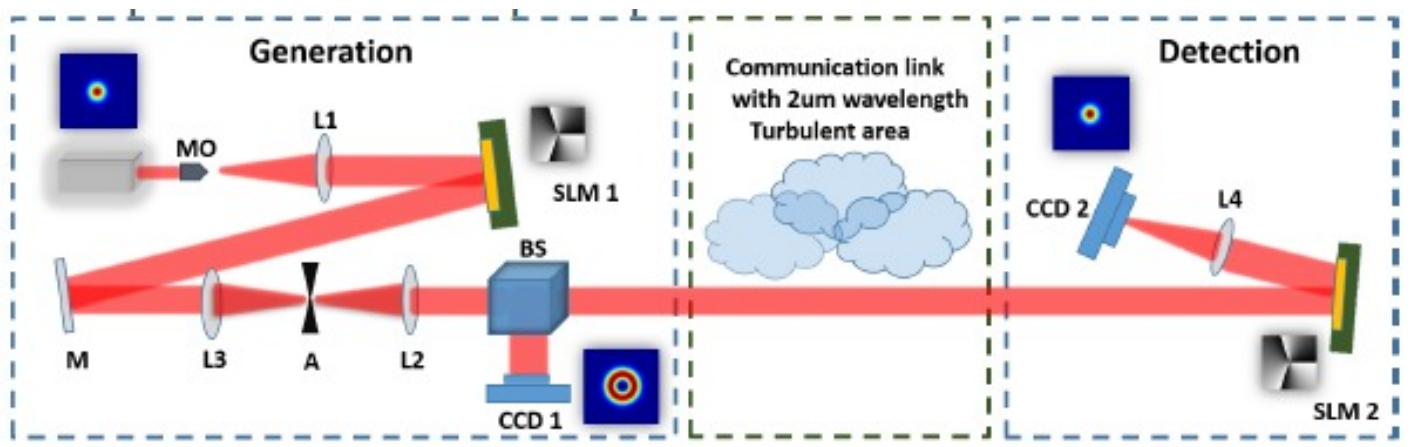

Figure 6. Experimental setup for Mid-IR long distance communication 
A long distance Mid-IR communication link will be established over 150 meters where information will be encoded using digital holography and also recovered using modal decomposition of the optical field. In addition to using LG modes, non-diffracting long-distance Bessel beam will be used as information carries. ${ }^{18-20}$

\section{REFERENCES}

[1] Richardson, D., Fini, J., and Nelson, L., "Space-division multiplexing in optical fibres," Nature Photonics 7(5), 354-362 (2013).

[2] Jono, T., Takayama, Y., Shiratama, K., Mase, I., Demelenne, B., Sodnik, Z., Bird, A., Toyoshima, M., Kunimori, H., Giggenbach, D., et al., "Overview of the inter-orbit and the orbit-to-ground laser communication demonstration by oicets," in [Lasers and Applications in Science and Engineering], 645702-645702, International Society for Optics and Photonics (2007).

[3] Doucet, M. A. and Panak, D. L., "Wireless fiber-coupled telecommunication systems based on atmospheric transmission of laser signals," (Feb. 19 2002). US Patent 6,348,986.

[4] Krenn, M., Fickler, R., Fink, M., Handsteiner, J., Malik, M., Scheidl, T., Ursin, R., and Zeilinger, A., "Communication with spatially modulated light through turbulent air across vienna," New Journal of Physics 16(11), 113028 (2014).

[5] Krenn, M., Handsteiner, J., Fink, M., Fickler, R., and Zeilinger, A., "Twisted photon entanglement through turbulent air across vienna," Proceedings of the National Academy of Sciences 112(46), 14197-14201 (2015).

[6] Gibson, G., Courtial, J., Padgett, M. J., Vasnetsov, M., Pasko, V., Barnett, S. M., and Franke-Arnold, S., "Free-space information transfer using light beams carrying orbital angular momentum," Optics Express 12(22), 5448-5456 (2004).

[7] Trichili, A., Salem, A. B., Dudley, A., Zghal, M., and Forbes, A., "Encoding information using laguerre gaussian modes over free space turbulence media," Optics Letters 41(13), 3086-3089 (2016).

[8] Trichili, A., Rosales-Guzmán, C., Dudley, A., Ndagano, B., Ben, S. A., Zghal, M., and Forbes, A., "Optical communication beyond orbital angular momentum.," Scientific reports 6, 27674 (2016).

[9] Forbes, A., Dudley, A., and McLaren, M., "Creation and detection of optical modes with spatial light modulators," Advances in Optics and Photonics 8(2), 200-227 (2016).

[10] Arrizón, V., Ruiz, U., Carrada, R., and González, L. A., "Pixelated phase computer holograms for the accurate encoding of scalar complex fields," JOSA A 24(11), 3500-3507 (2007).

[11] Goodman, J. W., [Introduction to Fourier optics], Roberts and Company Publishers (2005).

[12] Schulze, C., Naidoo, D., Flamm, D., Schmidt, O. A., Forbes, A., and Duparré, M., "Wavefront reconstruction by modal decomposition," Optics express 20(18), 19714-19725 (2012).

[13] Flamm, D., Naidoo, D., Schulze, C., Forbes, A., and Duparré, M., "Mode analysis with a spatial light modulator as a correlation filter," Optics letters 37(13), 2478-2480 (2012).

[14] Schulze, C., Ngcobo, S., Duparré, M., and Forbes, A., "Modal decomposition without a priori scale information," Optics express 20(25), 27866-27873 (2012).

[15] Ndagano, B., Sroor, H., McLaren, M., Rosales-Guzmán, C., and Forbes, A., "Beam quality measure for vector beams," Optics Letters 41(15), 3407-3410 (2016).

[16] Schulze, C., Dudley, A., Flamm, D., Duparré, M., and Forbes, A., "Measurement of the orbital angular momentum density of light by modal decomposition," New Journal of Physics 15(7), 073025 (2013).

[17] Andrews, L. C. and Phillips, R. L., [Laser beam propagation through random media], vol. 52, SPIE press Bellingham, WA (2005).

[18] Belyi, V., Forbes, A., Kazak, N., Khilo, N., and Ropot, P., "Bessel-like beams with z-dependent cone angles," Optics express 18(3), 1966-1973 (2010).

[19] Litvin, I. A., Mhlanga, T., and Forbes, A., "Digital generation of shape-invariant bessel-like beams," Optics express 23(6), 7312-7319 (2015).

[20] Litvin, I., Burger, L., and Forbes, A., "Self-healing of bessel-like beams with longitudinally dependent cone angles," Journal of Optics 17(10), 105614 (2015). 